\title{
Investigation on the interfacial properties of CNTs sized carbon fibres in epoxy resin using push-out method via nano-indentation technique
}

\author{
Zhenxue Zhang ${ }^{1 *}$, Xiaoying $\mathrm{Li}^{1}$, Hanshan Dong ${ }^{1}$, Simon Jestin ${ }^{2}$, Stefania Termine ${ }^{3}$, \\ Aikaterini-Flora Trompeta ${ }^{3}$, Andreia Araújo ${ }^{4}$, Raquel M. Santos ${ }^{4}$ and Costas Charitidis ${ }^{3}$ \\ ${ }^{1}$ School of Metallurgy and Materials, University of Birmingham, Edgbaston, Birmingham, UK \\ ${ }^{2} \mathrm{CANOE}$, Le Centre Technologique Nouvelle Aquitaine Composites \& Matériaux Avancés, Bât \\ CHEMINNOV - ENSCBP, 33600 Pessac, France \\ ${ }^{3}$ Research Lab of Advanced, Composites, Nanomaterials and Nanotechnology (R-NanoLab), School \\ of Chemical Engineering, National Technical University of Athens, GR15780, Athens, Greece \\ ${ }^{4}$ Institute of Science and Innovation in Mechanical and Industrial Engineering (INEGI), Materials and \\ Composite Structures unit, Porto, Portugal
}

\begin{abstract}
In this work, the carbon fibres (CFs) surfaces were modified via sizing and coated with a very thin layer of a complex formulation including carbon nanotubes (CNTs). A push-out method was developed based on nanoindentation to assess the interfacial shear strength of the fibre/matrix. The mechanical properties such as indentation hardness, reduced modulus, indentation displacement and indentation creep of the composite were evaluated by means of the Oliver-Pharr method. The critical load of different composites was measured and the interfacial shear strength (IFSS) was calculated to compare the effect of the CNTs concentration in the sizing solution. Wettability evaluation of the sized fibres was performed prior to nanoindentation to investigate the adhesion of the resin. After push-out testing, characterisation by optical microscopy/SEM was carried out to ratify the results. It was found sizing with a small amount of evenly distributed nano-inclusion on CFs can increase the interfacial shear strength but large amount of sizing could lead to a decrease of the interfacial bonding due to the agglomeration of CNTs on CFs.
\end{abstract}

Keywords: push-out, CNT, carbon fibre reinforced composite, nano-indentation, wettability

\section{Introduction}

A carbon fibre reinforced polymer (CFRP) composite consists of a continuous matrix (resin) which helps to keep the shape and CFs distributed within it to ensure the strength of materials. The interface transfers the load from the matrix to the fibres and, thus, it is responsible for the effect of "reinforcement". The interfacial properties of fibre and resin often influence significantly the performance of the composites, such as failure mode and fracture toughness.

* Corresponding author: z.zhang.1@bham.ac.uk 
Different approaches have been used to chemically or physically improve the bonding between the CFs and the matrix [1,2]. Simple mixing of CNTs with the polymer resin is the basic method for fabricating CFRPs doped with nanoparticles[3, 4]. However, the incorporation of increasing amounts of CNTs greatly increases the viscosity of the resin, while a non-uniform nanoparticle dispersion, acting as defect points, leads to a decrease of the mechanical performance of the material [5]. CNT-grafted CFs have been developed by directly growing or synthesizing CNTs on the surface of CFs, as for example through chemical vapour deposition, however, it is difficult to upscale the production [6-8]. Cheon used a complex esterification reaction of a coupling agent to introduce functional groups on the MWCNTs and the CFs through acid and flame treatments, respectively. MWCNTanchored CFs can improve the impact resistance and interlaminar shear strength of the composites [9]. Sizing was used to treat CFs by dispersing CNTs in agents of water-borne polycarbonate (WPC) and water-borne polyurethane (WPU). It was found small content of CNTs addition has a beneficial effect on the bonding between $\mathrm{CF}$ (CF) and polycarbonate (PC) [10]. In this paper, the CFs surfaces are modified via sizing - in which reinforcing CFs are coated with a very thin layer of a complex formulation including CNTs. This formulation aims at easy processing of the fibre but also at creating strong interfaces between the fibre and the matrix resin in the final composite.

In order to determine parameters of interfacial interaction between fibres and matrices, intensive work has been done to understand the influence of the interface on the mechanical behaviour of composites $[11,12]$. Matrix externally loaded and single fibre directly loaded methods are commonly used. Typically, push-out test is carried out directly on individual CF in the composite sample which result in quantitative values of the interfacial shear strength (IFSS) directly [13]. In the push-out test, the load is applied continuously on a single fibre using a specially designed indenter and a counter plate is used to support the thin disc sample. As the load increases, cracks are initiating and propagating along with the interface, producing a non-linear section. A complete debonding of the fibre is marked by a constant load with increased displacement corresponding to a critical load. The average interfacial shear strength (IFSS) at the fibre/matrix interface can be calculated by the critical load, the sheet thickness and the fibre diameter [14].

As a preliminary test, wettability assessment took place, in order to investigate the spreadability of the resin on the sized CFs bundles. The interfacial properties of sized CFs by CNTs and epoxy resin were investigated using the instrumental nano-indentation machine. The mechanical properties such as indentation hardness, reduced modulus, indentation displacement and indentation creep of the composite were evaluated by means of the OliverPharr method [15]. The critical load of different composites was measured and the IFSS was calculated to compare the effect of different amounts of CNTs in the sizing agent. After pushout, characterisations by OM/SEM were carried out to ratify the results.

\section{Experimental details}

Sizing is a surface coating process in which a reinforcing CF is coated with a very thin layer of a complex formulation. The sizing solution was obtained by mixing the commercial polyurethane dispersion (MICHELMAN HYDROSIZE U6-01) with the CNTs filler (CNT, Arkema C100 Graphistrength) dispersed by an anionic surfactant, sodium dodecyl benzenesulfonate (SDBS). Sizing content obtained is up to $2.2 \mathrm{wt} \%$ and nanofiller concentration in the sizing layer is between 1 and $10 \mathrm{wt} \%$ (Table 1). T700 SC $31 \mathrm{E} \mathrm{CFs}$ (T700) with a diameter of approximately $7 \mu \mathrm{m}$ from Toray were sized with these CNTs formulation containing emulsifiers, anti-static agents, lubricants as well as a polymeric coupling agent that promotes fibre-matrix interactions and thus creating strong interfaces 
between the fibre and the matrix resin in the composite. Fibre sizing was carried out continuously from spool to spool by immersing the T700 CF tow in the sizing bath containing the nanotube-polyurethane formulation followed by squeezing of the excess solution and subsequent drying at $200^{\circ} \mathrm{C}$ in an oven prior rewinding at $5 \mathrm{~m} / \mathrm{min}$. The blank fibre as reference (B1) corresponds to original polyurethane sizing.

Table 1. Detail of sizing of T700 CFs with CNTs in an epoxy resin composite

\begin{tabular}{|c|c|c|c|c|}
\hline Code & Sizing & Surfactant & \% sizing & \% filler in sizing \\
\hline B1 & Blank & - & 1.4 & 0 \\
\hline U1T1 & $1 \%$ CNT & SDBS & 1.4 & 1 \\
\hline U5T1 & $5 \%$ CNT & SDBS & 1.4 & 5 \\
\hline U10T1 & $10 \%$ CNT & SDBS & 2.2 & 10 \\
\hline
\end{tabular}

For the surface wettability assessment of the sized CFs, a prototype contact angle apparatus was used with high-resolution cameras. Specifically, an epoxy resin droplet was deposited on mounted CFs from an orifice of defined diameter $(\mathrm{d}=4 \pm 1 \mathrm{~mm})$. Video capturing from $\mathrm{t}_{0}$ (deposition time) to equivalence time $(\sim 90 \mathrm{sec})$ took place and then frames on specific time intervals $(10,20,40,80 \mathrm{sec})$ were selected to measure the droplets length to quantify the spreading time.

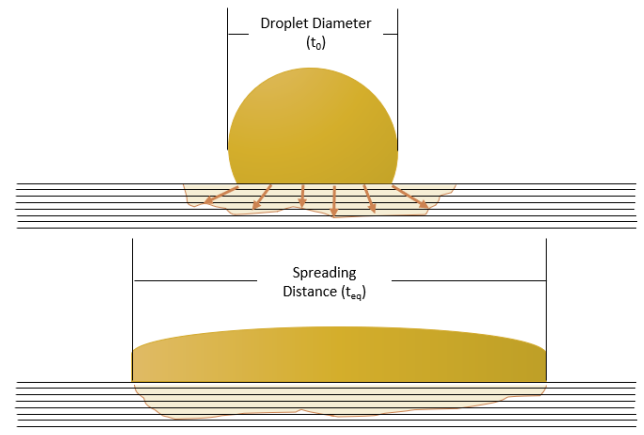

Fig. 1. Schematic representation of resin wettability measurement on CF bundle.

Epoxy Resin CETEPOX AM 3321 with hardener was used as a matrix for the preparation of the infused composites. After resin curing under vacuum, the composites plates were cut into coupons and polished. Thin discs were cut from the mounted composite with a thickness less than $1 \mathrm{~mm}$, and then the disc was broken by a sharp blade into small pieces (Fig.2a). The polished side of the small piece was attached to an aluminium stud and ground progressively by Grit 1200, 2500 and 4000 sandpapers to under $100 \mu \mathrm{m}$ thick, followed by polishing with colloidal silica suspension on a Struers MD-Chem napped cloth. The piece was then stuck to a homemade holder with laser machined $30 \mu \mathrm{m}$ wide grooves (Fig.2b). The fibres in the composite after push-out tests were observed via the onsite microscopy (Fig.2c) or by a JEOL7000 SEM after being unloaded from the holder.

A Micro Materials NanoTest system was used for the nanoindentation, push-out and creep testing. The experiments were conducted in an environmental enclosure controlled at $20.0 \pm 1.0^{\circ} \mathrm{C}$. Firstly, a suitable area and individual fibres were selected under optical microscopy (Fig.2c). All the samples were loaded from an initial load $10 \mu \mathrm{N}$ to a peak load (up to $100 \mathrm{mN}$ ) at a loading rate of $1 \mathrm{mN} / \mathrm{s}$. The test was normally carried out five or more times under same conditions. A specially made cone-shaped indenter was used in the pushout test and the maximum effective depth is about $2.2 \mu \mathrm{m}$ for a fibre of $7 \mu \mathrm{m}$ in diameter. The nanoindentation creep test was conducted at a load lower than the critical load. 


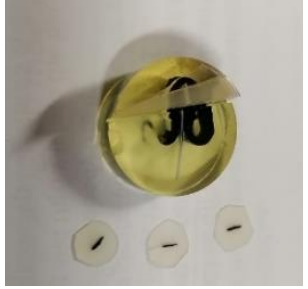

(a)

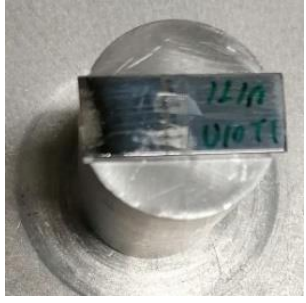

(b)

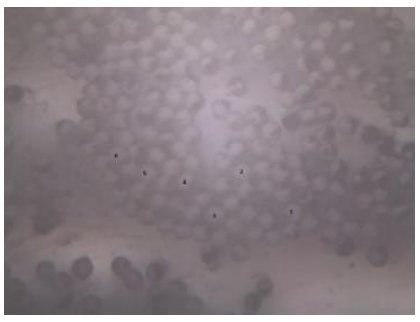

(c)

Fig. 2. (a) Composite specimen preparation, (b) set-up for push-out test, and (c) optical microscopy image of the sample U5T1

\section{Results and discussion}

\subsection{Wettability assessment of CNT-sized CFs}

The wettability results are shown in Fig.3. The length of the resin droplet was measured at the moment of contact with the CF bundle $\left(\mathrm{t}_{0}\right)$ and on the selected timeframes. Then, the average spreading rate was calculated for each CNT concentration of the sizing, considering a linear relationship between the increase of droplet diameter and time (Table 2). The sizing with $1 \%$ CNT presented the highest spreading rate, indicating higher wettability with the resin. The sized fibres with higher CNT concentrations showed very low spreading rate and no variance in the spreading length of the droplet. This is an indication that above $5 \%$ CNTs in the sizing formulation, the surface tension increases and burdens the fibre wettability.

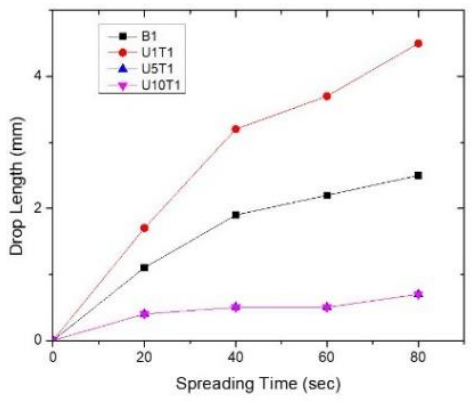

Table 2. Spreading rates of epoxy resin on CF bundles.

\begin{tabular}{|c|c|}
\hline Code & Spreading Rate $(\mathbf{m m} / \mathbf{s e c})$ \\
\hline B1 & 0.03 \\
\hline U1T1 & 0.06 \\
\hline U5T1 & 0.01 \\
\hline U10T1 & 0.01 \\
\hline
\end{tabular}

Fig.3. Wettability assessment on CF with CNTs based sizing formulation: dependence of wetting drop length and spreading time.

Table 3. Nano-hardness (H) and reduced elastic modulus (Er) of sized CFs and resin in the composite

\begin{tabular}{|c|c|c|c|c|c|}
\hline \multirow{2}{*}{$\begin{array}{c}\text { Sample } \\
\text { code }\end{array}$} & \multirow{2}{*}{ Detail } & \multicolumn{2}{|c|}{ CF } & \multicolumn{2}{c|}{ Resin } \\
\cline { 3 - 6 } & & $\mathrm{H}(\mathrm{GPa})$ & $\mathrm{Er}(\mathrm{GPa})$ & $\mathrm{H}(\mathrm{GPa})$ & $\operatorname{Er}(\mathrm{GPa})$ \\
\hline B1 & U6-01 Blank & $5.82 \pm 0.36$ & $43.06 \pm 8.98$ & $0.260 \pm 0.06$ & $5.62 \pm 0.20$ \\
\hline U1T1 & U6-01 - 1\%CNT & $5.24 \pm 0.45$ & $36.16 \pm 6.99$ & $0.264 \pm 0.05$ & $5.20 \pm 0.25$ \\
\hline U5T1 & U6-01 - 5\%CNT & $5.28 \pm 0.52$ & $45.30 \pm 9.04$ & $0.260 \pm 0.06$ & $5.12 \pm 0.33$ \\
\hline U10T1 & U6-01 - 10\%CNT & $5.25 \pm 0.42$ & $44.50 \pm 7.69$ & $0.229 \pm 0.04$ & $5.59 \pm 0.34$ \\
\hline
\end{tabular}




\subsection{Nano-hardness and reduced elastic modulus of the CFs and Resin}

Indentation tests were carried out on the CFs and the resin in the reinforced composite, to evaluate their mechanical properties separately, and the results are shown in Table 3. CFs have much higher nano-hardness and reduced modulus than the resin, which ensure the highstrength $\mathrm{CF}$ reinforce the relative lower strength resin to obtain the desired properties of composite materials (Fig. 4).

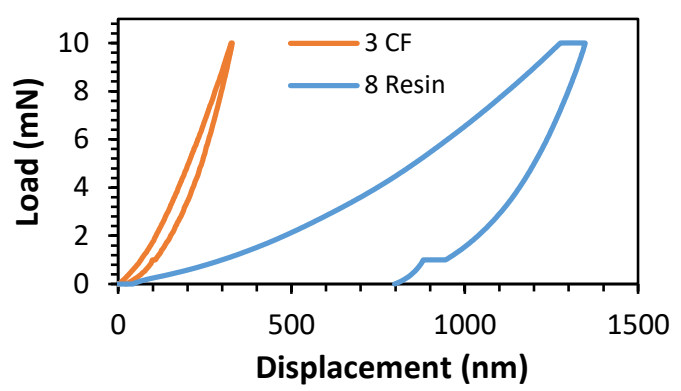

Fig. 4. Load vs displacement curves for typical CF and resin tests

\subsection{Push-out test}

In the push-out test (POT), the load was applied on a single fibre using the diamond indenter and a counter plate was used to support the thin disc sample. A complete debonding of the fibre is only possible for very thin specimens, with a thickness typically between 30-60 $\mu \mathrm{m}$. As shown in Fig.5b, a typical area was selected and individual fibres were identified. Then, a load was gradually applied to each location to a maximum value $(80 \mathrm{mN})$ which was greater than the critical load and decided experimentally. There was a short initial non-linear stage until a conformal contact was reached between the indenter, the specimen and the supporting plate especially, followed by a linear elastic region (Fig.5a). As the load increased to a certain level, a constant load with increased displacement was reached, which corresponds to the debonding of the fibre, and this is the critical load. The average interfacial shear strength (IFSS) at the fibre/matrix interface can be decided by the applied load, the fibre radius and the thickness of the specimen sheet [14]. The load vs displacement curves of the seven CFs can be seen in Fig.5a. Observations on the disc by on-site optical microscopy can confirm that there were $6 \mathrm{CFs}$ and one location on resin was selected. Corresponding to the curves and the indentations analysis (Fig.5c/d), fibres 1, 2, 3 and 4 are located on the top of the grooves and can be used to calculate the interfacial shear strength. However, fibre 5 strongly interfered with the neighbouring fibres, and fibre 6 was located outside the groove which cannot be used. Location 7 was used as a reference for the resin. Therefore, fibres 5, 6 and 7 were not included in the statistics. In short, for the sample U1T1, with $1 \%$ CNT modified U601, the corresponding critical load and calculated IFSS are shown in Fig.5e. For the sample U5T1, with 5\%CNT modified U601, the average critical load is $53.0 \pm 9.1 \mathrm{MPa}$ and the calculated IFSS is about $78.1 \pm 14.9 \mathrm{mN}$, for a $31 \mu \mathrm{m}$ thick disc. For the sample U10T1, with $10 \%$ CNT modified U601, the average IFSS is $68.1 \pm 7.2 \mathrm{MPa}$ for a $30 \mu \mathrm{m}$ thick specimen. The critical load for sample B1 (U601-Blank) is about $42.1 \pm 5.1 \mathrm{mN}$ and IFSS is $76.2 \pm 12.1 \mathrm{MPa}$, measured for a $25 \mu \mathrm{m}$ thick location. 


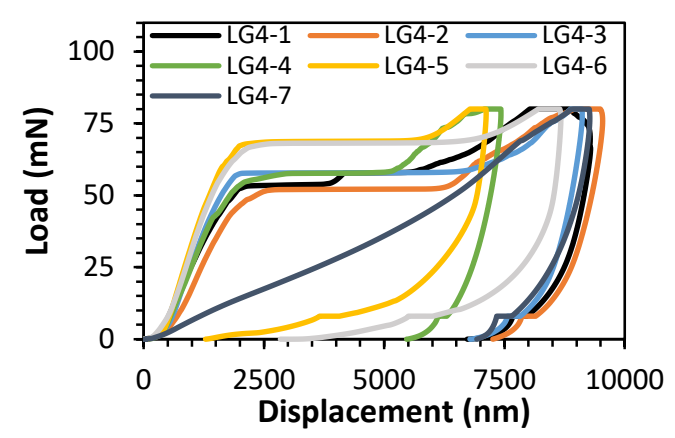

(a)

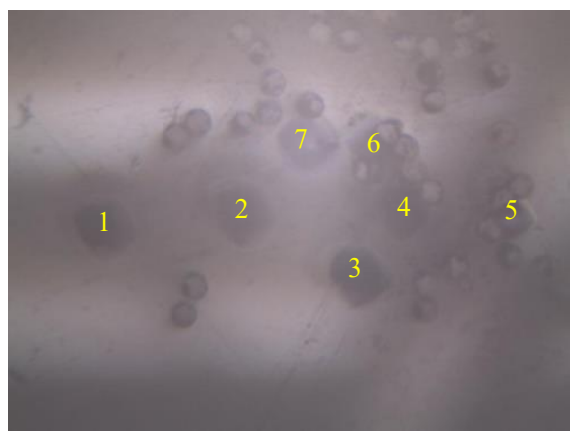

(c)

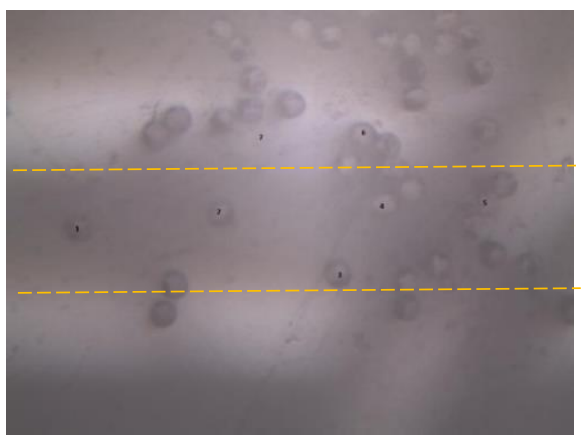

(b)

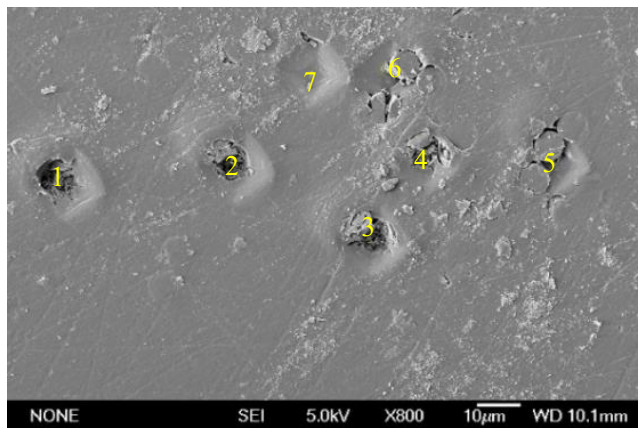

(d)

\begin{tabular}{|c|c|c|}
\hline & Critical load (mN) & IFSS (MPa) \\
\hline LG4-1 & 53.6 & 87.1 \\
\hline LG4-2 & 52.1 & 84.6 \\
\hline LG4-3 & 57.7 & 93.7 \\
\hline LG4-4 & 55.8 & 90.6 \\
\hline Average & $54.8 \pm 2.9$ & $89.0 \pm 4.7$ \\
\hline
\end{tabular}

(e)

Fig. 5. Sample U1T1 epoxy composite: (a) load vs displacement curves (b) selected test area and CFs, observations after POT by (c) OM and (d) SEM, (e) the critical load and IFSS results

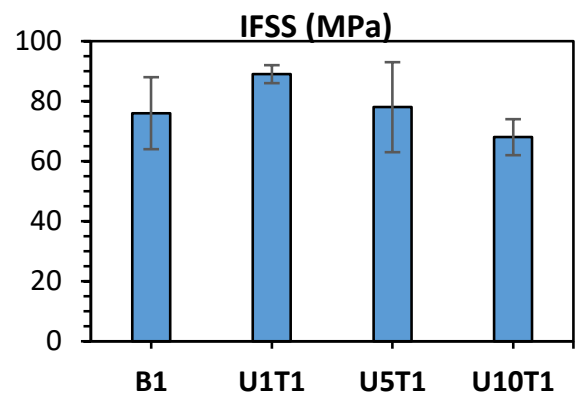

Fig. 6. Comparison of the interfacial shear strength of the sized CFs reinforced epoxy resin with the blank one 
Fig. 6 presents a comparison of the IFSS results obtained for the reinforced epoxy resin with different sized CFs. As it can be observed, the IFSS increased for a lower $\%$ of CNTs addition (U1T1) and continues to decrease with the increasing amount of nanoparticles dispersed in the sizing solutions. For instance, the sized CF with $1 \%$ CNT had an $18 \%$ IFSS increase when compared to the blank, while for sample U10T1, with $10 \%$ of CNTs, the IFSS was reduced by $10 \%$. Nevertheless, it should be noted the higher error associated with the results for the Blank samples. Further examinations on the CFs after sizing indicated that with higher amounts of CNTs in the sizing solution, such as for U5T1 and U10T1, agglomeration can be found on the CFs surface (Fig. 7b). For the samples U1T1 no agglomerated CNTs were found on the sized CFs surface. This is in agreement with Liu's report that the addition of CNTs has a beneficial effect on the interfacial properties of composites when the content of CNTs is low, such as around $0.1 \mathrm{wt} \%$. High amounts of CNTs causes agglomeration, which is unfavourable for the improvement in interfacial properties [10]. In summary, the sizing agents play a key role to enforce the compatibility between the CFs and the resin in the composite, and smaller amounts of CNTs can effectively prevent interfacial slip, thus significantly improving the interfacial properties in CFRPs. Large amounts of nano-fillers can negatively influence the interface between the CFs and the matrix due to the presence of agglomerates that may act as defects and stress concentration points.

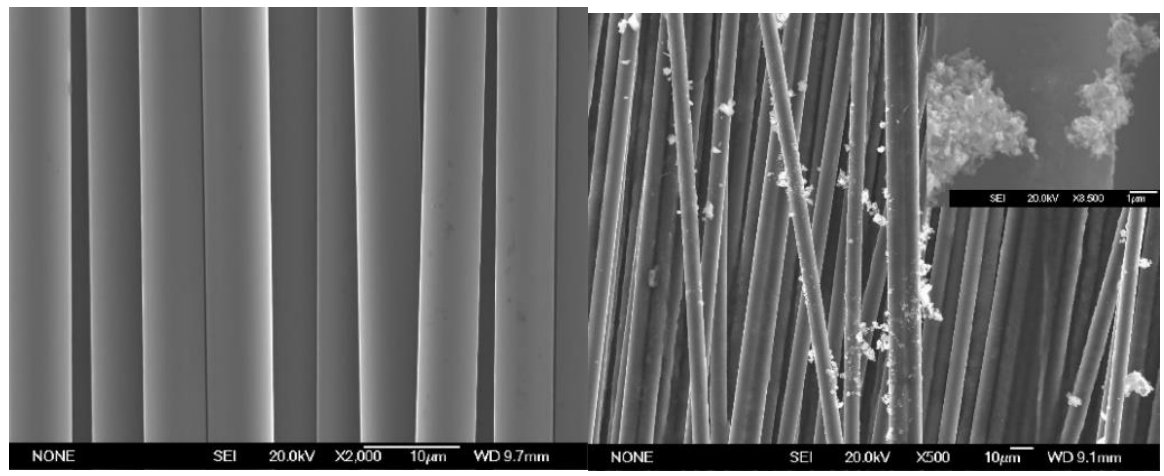

(a)

(b)

Fig.7. (a) T700 CFs without sizing (b) CFs with 5\% CNTs in sizing agent (U5T1) and the CNTs agglomerate on the $\mathrm{CF}$ (inset)

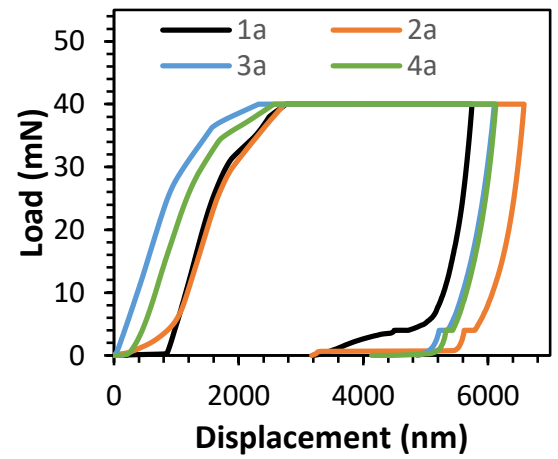

(a)

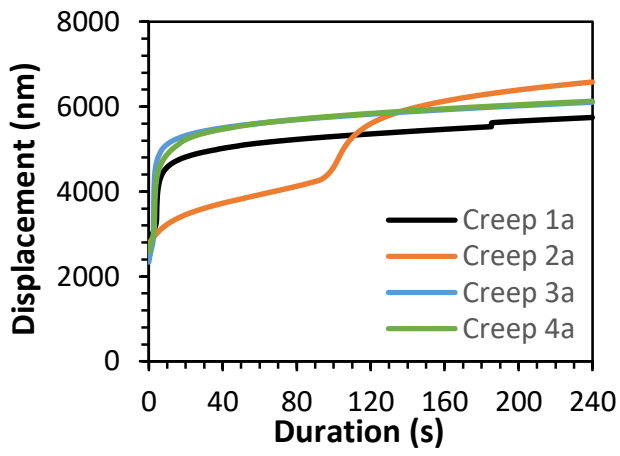

(b)

Fig.8. Sample of U10T1 epoxy composite: (a) load vs displacement curves, (b) displacement of the $\mathrm{CF}$ at holding period 


\subsection{Creep behaviour of the CF in a composite}

For sample U10T1, a load of $40 \mathrm{mN}$ and above is needed to push a CF out, so $40 \mathrm{mN}$ was used to observe the creep behaviour of the CF in a thin disc. The displacement against the loading and the unloading and the dwell time (240s) were plotted in Fig. 8, where a large displacement can be found for all the four CFs selected (Fig.8a). It was found that a creep behaviour occurred at the dwelling period which is indicated by the sudden hike of displacement as seen in Fig.8b.

\section{Conclusions}

In this work, CFs surfaces were modified with a very thin layer via sizing by a complex formulation including different amounts of CNTs, which aims at creating strong interfaces between the fibre and the matrix in the composite. A push-out method based on nanoindentation was used to measure the critical load, and thus to calculate the interfacial shear strength (IFSS) to compare the effect of the varied sizing amount of CNTs. It was found sizing with a small amount of evenly distributed CNTs on CFs can increase IFSS by $18 \%$, but a large amount of sizing could lead to a $10 \%$ decrease of the interfacial bonding due to the agglomeration of CNTs on the CFs. Wettability testing with epoxy resin, confirmed the above results, indicating that the CFs sized with $1 \%$ CNTs, enhance the spreading of the resin, thus ensure the processability of the fibres during infusion and lead to increased adhesion of the CFs with the polymeric matrix.

\section{Acknowledgments}

This work was supported by the EU H2020 Project 'Smart by Design and Intelligent by Architecture for turbine blade fan and structural components systems' (SMARTFAN) under Grant no. 760779.

\section{References}

[1] P.Hung, K.Lau, B.Fox, N.Hameed, J.Lee, D.Hui, Compos.-B: Eng. 133 (2018) 240-257.

[2] A.Khan, D.Dragatogiannis, P.Jagdale, M.Rovere, C.Rosso, A.Tagliaferro, C.Charitidis, EXPRESS polym. lett. 15(4) (2021) 361-374.

[3] B.Salahuddin, S.Faisal, T.Baigh, M.Alghamdi, M.S. Islam, B. Song, X. Zhang, S. Gao, S. Aziz, Polymers 13(16) (2021).

[4] A.Trompeta, E.Koumoulos, S.Stavropoulos, T.Velmachos, G.Psarras, C.Charitidis, Aerospace 6(1) (2019).

[5] T.Li, M.Li, Y.Gu, S.Wang, Q.Li, Z.Zhang, Compos. Sci. Technol. 166 (2018) 176-182.

[6] S.Sharma, S.Lakkad, Compos. -A: Appl. Sci. Manuf. 42(1) (2011) 8-15.

[7] S.Termine, A.Trompeta, D.Dragatogiannis, C.Charitidis, MATEC Web Conf. 304 (2019).

[8] F.An, C.Lu, Y.Li, J.Guo, X.Lu, H.Lu, S.He, Y.Yang, Mater. Des. 33 (2012) 197-202.

[9] J.Cheon, M.Kim, Compos. -B: Eng. 217 (2021) 108872.

[10] Y.Liu, L.Li, J.Wang, Y.Fei, N. Liu, G.Wu, New Carbon Mater. 36(3) (2021) 639-648.

[11] S.Zhandarov, E.Mäder, Compos. Sci. Technol. 65(1) (2005) 149-160.

[12] S.Huang, Q.Fu, L.Yan, B.Kasal, J. Mater. Res. Technol. 13 (2021) 1441-1484.

[13] Z.Zhang, S.Gallo, X.Li, H.Dong, D.Dragatogiannis, C.Charitidis, Polym. Test. 80 (2019) 106091.

[14] S.Corujeira Gallo, X.Li, Z.Zhang, C.Charitidis, H.Dong, Compos.-A: Appl. Sci. Manuf. 112 (2018) 178-185.

[15] H.Lu, B.Wang, J.Ma, G.Huang, H.Viswanathan, Mech. Time-Depen. Mater. 7(3) (2003) 189-207. 